\title{
Molar Electron Mass and the Basics of TOE
}

\author{
U. V. S. Seshavatharam ${ }^{1, *}$, S. Lakshminarayana ${ }^{2}$ \\ ${ }^{1}$ Honorary faculty, I-SERVE, Alak apuri, Hyderabad-35, AP, India \\ ${ }^{2}$ Dept. of Nuclear Physics, Andhra University, Visakhapatnam-03, AP, India
}

\begin{abstract}
The subject of unification is very interesting and very complicated. Implementing the gravitational constant in atomic and nuclear physics is a challenging task. Still string theory and super gravity are not in a position to bring down the Planck scale to the nuclear scale. In this connection, considering the 'mo lar electron mass' an attempt is made to study the four fundamental interactions in a unified manner.
\end{abstract}

Keywords Gravity, Avogadro Number, Integral Charge Quark SUSY, Quark Fermion, Quark Boson, Quark Baryon, Quark Meson, Higgs Fermion, Higgs Boson, Strong Coupling Constant, SEMF Energy Coefficients, Electron and the Nucleon'S Magnetic Moments, Gram Mole

\section{Introduction}

String theory and Super gravity are in the race of developing the real TOE. It is very interesting to implement the gravitational constant in atomic and nuclear physics. With gravity - interpreting the known atomic and nuclear phenomena, fitting and estimating the elementary particle rest masses and their coupling strengths is a very hard and sensitive job. Einstein spent most of his life in unifying gravity and electromagnetism. But by that time, strong interaction was in its budding stage. Still string theory and super gravity are not in a position to bring down the Planck scale to the nuclear scale. In this critical situation, by considering the "molar electron mass", an attempt is made to understand the secrets of unification.

On 21 December 2011 a new meson of rest energy $10.530 \pm 0.005 \mathrm{GeV}$ was detected in CERN - LHC and the ATLAS detector. This new meson, known as $\chi_{b}(3 p)$, consists of two parts - an elementary particle known as a 'beauty' quark and its opposite antiquark, which are bound together by a 'strong force'[1]. Its existence was predicted in our published paper[2] : page-278, table-16, last row, last column. Before going further, authors request the interested readers to please go through the two published papers[2] and[3]. This paper is a combined and unified version of the published papers $[2,3]$ and proceedings of the DAE symposium on nuclear physics 2011 , India [4,5]. Please note that in our previous paper[2] it was suggested that: $W$ boson is the super symmetric boson of the top quark fermion and the charged Higgs boson pair generates the neutralized $Z$ boson. It was also suggested that $[3,5]$ Higgs charged boson

\footnotetext{
* Corresponding author:

seshavatharam.uvs@gmail.com (U. V. S. Seshavatharam)

Published online at http://journal.sapub.org/jnpp

Copyright (C) 2012 Scientific \& Academic Publishing. All Rights Reserved
}

and $W$ boson couples together to form a neutral boson of rest energy $126 \mathrm{GeV}$. Its existence was detected and is under open discussion[6,7]. Another interesting idea is: $W$ boson pair generates a neutral boson of rest energy 161 $\mathrm{GeV}$. This is our prediction and needs to be verified.

\section{Key Assumptions in Unification}

The subject of unification is very interesting and very complicated. By implementing the Avogadro number $N$ as a scaling factor in unification program, one can probe the constructional secrets of elementary particles. The Planck's quantum theory of light, thermodynamics of stars, black holes and cosmology totally depends upon the famous Boltzmann constant $k_{B}$ which in turn depends on the Avogadro number[8]. Thus it can be suggested that, Avogadro number is more fundamental and characteristic than the Boltzmann constant and indirectly plays a crucial role in the formulation of the quantum theory of radiation. In this connection it is noticed that, 'molar electron mass' plays a very interesting role in nuclear and particle physics. With the following four assumptions- the string theory[911], super gravity[12,13] and strong gravity[14-22] can be studied in a unified manner.

Assumption-1: Nucleon behaves as if it constitutes molar electron mass. (Or) Molar electron mass $\left(N . m_{e}\right)$ plays a crucial role in nuclear and particle physics.

Assumption-2: The key conceptual link that connects the gravitational and non-gravitational forces is - the classical force limit

$$
F_{C} \cong\left(\frac{c^{4}}{G}\right) \cong 1.21026 \times 10^{44} \text { newton }
$$

It can be considered as the upper limit of the string tension. In its inverse form it appears in Einstein's theory of 
gravitation as $\frac{8 \pi G}{c^{4}}$. It has multiple applications in Black hole physics and Planck scale physics[23]. It has to be measured either from the experiments or from the cosmic and astronomical observations.

Assumption-3: Ratio of 'c lassical force limit $\left(F_{C}\right)$ ' and 'weak force magnitude $\left(F_{W}\right)$, is $N^{2}$ where $N$ is a large number close to the Avogadro number.

$$
\frac{F_{C}}{F_{W}} \cong N^{2} \cong \frac{\text { upper limit of classical force }}{\text { nuclear weak force magnitude }}
$$

Thus the proposed weak force magnitude is $F_{W} \cong \frac{c^{4}}{N^{2} G} \cong 3.33715 \times 10^{-4}$ newton and can be considered as the characteristic nuclear weak string tension. It can be measured in the particle accelerators.

Assumption-4: In modified quark SUSY, if $Q_{f}$ is the mass of quark fermion and $Q_{b}$ is the mass of quark boson, then

$$
\frac{Q_{f}}{Q_{b}} \cong \Psi \cong 2.262706
$$

and $\left(1-\frac{1}{\Psi}\right) Q_{f}$ represents the effective fermion mass. The number $\Psi$ can be fitted with the following empirical relation $\Psi^{2} \ln \left(1+\sin ^{2} \theta_{W}\right) \cong 1$.

\section{Basic Ideas in 'Modified' Quark Super Symmetry}

Till today there is no reason for the question: why there exists 6 individual quarks? Till today no experiment reported a free fractional charge quark. Authors humble opinion is nuclear charge (either positive or negative) constitutes 6 different flavours and each flavour holds certain mass. Charged flavour can be called as a quark. It is neither a fermion nor a boson. A fermion is a container for different charges, a charge is a container for different flavours and each flavour is a container for certain matter. If charged matter rests in a fermionic container it is a fermion and if charged matter rests in a bosonic container it is a boson. The fundamental questions to be answered are : what is a charge? why and how opposite charges attracts each other? why and how there exists a fermion? and why and how there exists a boson? Here interesting thing is that if 6 flavours are existing with 6 different masses then a single charge can have one or two or more flavours simultaneously. Since charge is a common property, mass of the multiple flavour charge seems to be the geometric mean of the mass of each flavour. If charge with flavour is called as a quark then charge with multi flavours can be called as a hybrid quark. Hybrid quark generates a multi flavour baryon. It is a property of the strong interaction space - time - charge. This is just like different tastes or different smells of matter. Important consequence of this idea is that-for generating a baryon there is no need to couple 3 fractional charge quarks.

1. There exists nature friendly integral charge quark fermions.

2. For every integral charge quark fermion there exists a corresponding integral charge quark boson. Quark fermion and quark bos on mass ratio is close to 2.2627.

3. There exists integral charged massive quark fermigluons and integral charged massive quark boso-gluons. (Fermi-gluon means massive gluons having fermion behaviour and boso-gluon means massive gluons having boson behaviour. Quark femi-gluon can be called as the 'quark baryon' and quark boso-gluon can be called as 'quark meson').

4. Quark fermi-gluon or quark baryon masses can be expressed as $Q_{F} c^{2} \cong 0.2314\left[M_{H f}^{2} \times Q_{f}\right]^{\frac{1}{3}} c^{2}$ and Quark boso-gluon or quark meson masses can be expressed as $Q_{M} c^{2} \cong 0.2314\left[M_{H b}^{2} \times Q_{b}\right]^{\frac{1}{3}} c^{2}$ where $Q_{f}$ and $Q_{b}$ are the rest masses of quark fermion and quark boson respectively and $M_{H f}$ and $M_{H b}$ are the Higgs charged fermion and Higgs charged boson respectively.

5. $Q_{e f} \cong Q_{f}-Q_{b} \cong\left(1-\frac{1}{\Psi}\right) Q_{f}$ acts as the effective quark fermion. Effective quark baryon mass can be expressed as $Q_{E} c^{2} \cong 0.2314\left[M_{H f}^{2} \times Q_{e f}\right]^{\frac{1}{3}} c^{2}$. These effective quark baryons play a vital role in fitting the unstable baryon masses. Quark meson masses play a vital role in fitting the unstable meson masses.

6. Characteristic nuclear fermion is $938.272 \mathrm{MeV}$ and its corresponding nuclear boson is $\frac{938.272}{\Psi} \cong 414.67 \mathrm{MeV}$. This boson couples with the light quark bosons or light quark mesons and generates neutral ground states. Thus it is the mother of presently believed strange mesons like 493, 548, $1020 \mathrm{MeV}$ and 783, $890 \mathrm{MeV}$ etc.

7. Charged ground state baryon rest energy is $\left(Q_{E 1} Q_{E 2}\right)^{\frac{1}{2}} c^{2} \quad$ or $\left(Q_{E 1} Q_{E 2}^{2}\right)^{\frac{1}{3}} c^{2} \quad$ or $\quad\left(Q_{E 1} Q_{E 2} Q_{E 3}\right)^{\frac{1}{3}} c^{2}$ where $Q_{E 1}, Q_{E 2}$, and $Q_{E 3}$ represents any three effective quark baryons. Integral charge light quark bosons' in one or two numbers couples with the ground or excited effective quark baryons and generates doublets and triplets. This is just like 'absorption of photons by the electron'.

$$
\begin{aligned}
& \text { 8. Rest energy of nucleon is close to } \\
& \left(\frac{2 U_{F} D_{F}}{U_{F}+D_{F}}\right) c^{2} \cong 940.02 \mathrm{MeV} \text { and nucleon rest energy } \\
& \text { difference } \quad \text { is } \quad \text { close } \\
& \left(m_{n}-m_{p}\right) c^{2} \cong \sin ^{2} \theta_{W} \cdot\left(\frac{2 U_{f} D_{f}}{U_{f}+D_{f}}\right) c^{2} \cong 1.29623 \mathrm{MeV}
\end{aligned}
$$


9. Only oppositely charged quark mesons couples together to form a neutral meson. No two quark fermions couples together to form a meson. Neutral ground state meson rest energy is close to $\left(Q_{M 1}+Q_{M 2}\right) c^{2}$ where $Q_{M 1}$ and $Q_{M 2}$ represents any two quark mesons.

10. Fine rotational levels of any ground state energy $m_{x} c^{2}$ can be expressed as, if $\mathrm{n}=1,2,3 \ldots$, and $I=n(n+1)$, $\left(m c^{2}\right)_{I} \cong[I]^{\frac{1}{4}} m_{x} c^{2}$ and $\left(m c^{2}\right)_{I / 2} \cong[I / 2]^{\frac{1}{4}} m_{x} c^{2}$. Super fine rotational levels can be obtained as $\left(m c^{2}\right)_{I} \cong[I]^{\frac{1}{12}} m_{x} c^{2}$ and $\left(m c^{2}\right)_{I / 2} \cong[I / 2]^{\frac{1}{12}} m_{x} c^{2}$.

\subsection{The Lepton-quark Mass Generator}

With its earlier defined magnitude[2] and in the recently published paper[3] it was defined that

$$
X_{E} \cong \sqrt{\frac{4 \pi \epsilon_{0}\left(N^{2} G\right) m_{e}^{2}}{e^{2}}} \cong 295.0606338
$$

where $N$ is the Avogadro number, $G$ is the gravitational constant and $m_{e}$ is the rest mass of electron. It can be called as the lepton-quark-nucleon mass generator. It plays a very interesting role in nuclear and particle physics. Using this number leptons, quarks and nucleon rest masses can be fitted[2]. It can be expressed as

$$
X_{E} \cong \sqrt{\frac{4 \pi \epsilon_{0} G\left(N m_{e}\right)^{2}}{e^{2}}} \cong 295.0606338
$$

Weak coupling angle was defined as

$$
\frac{m_{u} c^{2}}{m_{d} c^{2}} \cong \sin \theta_{W} \cong \frac{1}{X_{E} \alpha} \cong 0.464433353
$$

where $m_{u}$ is the rest mass of up quark, $m_{d}$ is the rest mass of down quark and $\sin \theta_{W}$ is the weak coupling angle. In the modified SUSY, the fermion and boson mass ratio $\Psi$ can be fitted in the following way.

$$
\Psi^{2} \ln \left(1+\sin ^{2} \theta_{W}\right) \cong 1
$$

Thus $\Psi \cong 2.262706$. If $m_{f}$ is the mass of fermion and $m_{b}$ is the mass of its corresponding boson then

$$
m_{b} \cong \frac{m_{f}}{\Psi}
$$

With this idea super symmetry can be observed in the strong interactions[2] and can also be observed in the electroweak interactions [3].

\subsection{To fit the Muon and Tau Rest Masses}

Using $X_{E}$ charged muon and tau masses[3,24] were fitted in the following way.

$$
m_{l} c^{2} \approx \frac{2}{3}\left[a_{c}^{3}+\left(n^{2} X_{E}\right)^{n} a_{a}^{3}\right]^{\frac{1}{3}}
$$

where $a_{c}$ and $a_{a}$ are the coulombic and asymmetric energy coefficients of the semi empirical mass formula and $\mathrm{n}=0,1,2$. This is an approximate relation. Qualitatively this expression is connected with $\beta$ decay. Accuracy can be improved with the following relation.

$$
\text { If } \begin{gathered}
\mathrm{E}_{\mathrm{W}} \cong \sqrt{\frac{\mathrm{e}^{2} \mathrm{~F}_{\mathrm{W}}}{4 \pi \epsilon_{0}}} \cong 1.731843735 \times 10^{-3} \mathrm{MeV} \\
m_{l} c^{2} \cong\left[X_{E}^{3}+\left(n^{2} X_{E}\right)^{n} \sqrt{N}\right]^{\frac{1}{3}} E_{W}
\end{gathered}
$$

where $n=0,1,2$. If it is true that weak decay is due to weak nuclear force, then $\left(\frac{1}{N^{2}}\right) \frac{c^{4}}{G} \cong F_{W}$ can be considered as the characteristic weak force magnitude. Please refer the published papers for the mystery of electro weak bosons and the Higgs boson[2,3]. Please see table-1.

Table 1. To fit the muon and tau rest masses

\begin{tabular}{|c|c|c|}
\hline $\mathrm{n}$ & $\begin{array}{c}\text { Obt. Lep. energy } \\
(\mathrm{MeV})\end{array}$ & Exp. Lep. energy (MeV) \\
\hline 0 & Defined & $0.510998910(13)$ \\
\hline 1 & 105.951 & $105.6583668(38)$ \\
\hline 2 & 1777.384 & $1776.99(29)$ \\
\hline
\end{tabular}

\subsection{To correlate the Electron, Muon, Proton and the Charged Pion Rest Masses}

From the above table-1, if $m_{\mu} c^{2} \cong 105.95 \mathrm{MeV}$, surprisingly it is noticed that,

$$
m_{p} c^{2} \cong \frac{1}{\alpha} \cdot\left(\sqrt{m_{\mu} m_{e}}-m_{e}\right) \cong 938.29 \mathrm{MeV}
$$

Based on the proposed SUSY, it is also noticed that

$$
\left(m_{\pi} c^{2}\right)^{ \pm} \cong \frac{1}{\Psi} \cdot \sqrt{m_{\mu} m_{p}} \cong 139.34 \mathrm{MeV}
$$

These two obtained mass units can be compared with the proton and the charged pion rest masses respectively. In a unified scheme these interesting observations cannot be ignored.

\subsection{Nucle ons, Up \& Down Quarks and the Strong Coupling Constant}

It our earlier published papers[2,3] it was also defined that

$$
\frac{m_{u} c^{2}}{m_{e} c^{2}} \cong e^{X_{E} \alpha}
$$

where $m_{u}$ is the up quark rest mass and $m_{d}$ is the down quark rest mass respectively. In our earlier papers, suggested up quark mass is $4.4 \mathrm{MeV}$ and down quark mass is $9.476 \mathrm{MeV}$. With these magnitudes it is noticed that,

$$
\left(m_{n}-m_{p}\right) c^{2} \cong \ln \left(\frac{\sqrt{m_{u} m_{d}}}{m_{e}}\right) m_{e} c^{2}
$$

Here lhs $=1.2933 \mathrm{MeV}$ and rhs $=1.2963 \mathrm{MeV} . \mathrm{It}$ is also noticed that 


$$
\left(\frac{\sqrt{m_{u} m_{d}}}{m_{e}}\right) \cong \frac{1}{2} \sqrt{\frac{G\left(N . m_{e}\right)^{2}}{\hbar c}} \cong 12.60271
$$

With reference to the strong coupling constant $\alpha_{s}$ - it is also noticed that[24],

$$
\begin{aligned}
& \left(\frac{1}{\alpha}+\frac{1}{\alpha_{s}}\right) \sqrt{m_{u} m_{d}} c^{2} \cong 940 \mathrm{MeV} \\
& \frac{\sqrt{m_{u} m_{d}} c^{2}}{\left(m_{n}-m_{p}\right) c^{2}} \cong \ln \left(\frac{1}{\alpha}+\frac{1}{\alpha_{s}}\right)
\end{aligned}
$$

\subsection{To Fit the Strong Coupling Constant}

The strong coupling constant $\alpha_{s}$ is a fundamental parameter of the Standard Model. It plays a more central role in the QCD analysis of parton densities in the moment space. QCD does not predict the actual value of $\alpha_{s}$, however it definitely predicts the functional form of energy dependence $\alpha_{s}$. The value of $\alpha_{s}$, at given energy or momentum transfer scale, must be obtained from experiment. Determining $\alpha_{s}$ at a specific energy scale is therefore a fundamental measurement, to be compared with measurements of the electromagnetic coupling $\alpha$, of the elementary electric charge, or of the gravitational constant. Considering perturbative QCD calculations from threshold corrections, its recent obtained value at $\mathrm{N}^{3} \mathrm{LO}$ [25] is $\alpha_{s} \cong 0.1139 \pm 0.0020$. At lower side $\alpha_{s} \cong 0.1139-0.002=0.1119$ and at higher side $\alpha_{s} \cong 0.1139+0.002=0.1159$. It can be fitted or defined in the following way.

$$
X_{S} \cong \frac{1}{\alpha_{s}} \cong \ln \sqrt{\frac{4 \pi \epsilon_{0} G\left(N . m_{e}\right)^{2}}{e^{2}}}+\ln \sqrt{\frac{G\left(N . m_{e}\right)^{2}}{\hbar c}}
$$

Thus $X_{S} \cong 8.914239916$.

$$
\text { simply, } \frac{1}{\alpha_{\mathrm{s}}} \cong \mathrm{X}_{\mathrm{S}} \cong \ln \left(\mathrm{X}_{\mathrm{E}}^{2} \sqrt{\alpha}\right) \cong \frac{1}{0.112180063}
$$

This proposed value numerically can be compared with the current estimates of the $\alpha_{s}$. It is true that the proposed definition is conceptually not matching with the current definitions of the strong coupling constant. But the proposed definition considers all the fundamental gravitational and non-gravitational physical constants in a unified manner. This proposal can be given a chance. With this magnitude it is noticed that

$$
\begin{aligned}
& m_{n} c^{2} \cong M_{0} c^{2}-\frac{m_{u}}{m_{d}}\left(\frac{2 m_{u} m_{d}}{m_{u}+m_{d}}\right) c^{2} \cong 939.6 \mathrm{MeV} \\
& m_{p} c^{2} \cong M_{0} c^{2}-\sqrt{\frac{m_{u}}{m_{d}}}\left(\frac{2 m_{u} m_{d}}{m_{u}+m_{d}}\right) c^{2} \cong 938.30 \mathrm{MeV}
\end{aligned}
$$

where $M_{0} c^{2}=\left(\frac{1}{\alpha}+\frac{1}{\alpha_{s}}\right) \sqrt{m_{u} m_{d}} c^{2} \cong 942.393 \mathrm{MeV}$.

\section{Integral Charge Quark Fermions and their SUSY Bosons}

In the previous papers authors suggested that up, strange and bottom quarks are in geometric series. Similarly down, charm and top quarks are in another geometric series. Obtained quark fermion masses can be compared with the current estimates[24]. Up and down fermion masses can be given as

$u_{f} c^{2} \cong e^{\alpha X_{E}} \times m_{e} c^{2} \cong 4.4 \mathrm{MeV}$
where $\quad X_{E} \cong \sqrt{\frac{4 \pi \varepsilon_{0} G\left(N . m_{e}\right)^{2}}{e^{2}}} \cong 295.0606338$ and $\alpha$ is the fine structure ratio.

$$
d_{f} c^{2} \cong \alpha X_{E} \times u_{f} c^{2} \cong 9.4755 \mathrm{MeV}
$$

Here, $m_{e} c^{2}=$ rest energy of electron, $\alpha=$ fine structure ratio,$X_{E}=$ proposed lepton mass generator. It is very interesting to note that

$$
\frac{\text { Down fermion mass }}{\text { Up fermion mass }} \cong \frac{d_{f}}{u_{f}} \cong \alpha X_{E} \cong \frac{1}{\sin \theta_{W}}
$$

In this way $\sin \theta_{W}$ can be related with up and down quark mass ratio. Proposed USB geo metric ratio is

$$
g_{U} \cong\left[\alpha X_{E} \frac{\alpha X_{E}+1}{\alpha X_{E}-1}\right]^{2} \cong 34.66294
$$

If DCT series is the second generation series, its geometric ratio is

$$
\begin{gathered}
g_{D} \cong\left[2 \alpha X_{E} \frac{\alpha X_{E}+1}{\alpha X_{E}-1}\right]^{2} \cong 138.651754 \\
\frac{g_{D}}{g_{U}} \cong \frac{\text { DCT geometric ratio }}{\text { USB geometric ratio }} \cong 4 .
\end{gathered}
$$

Quark boson mass $=Q_{b} \cong \frac{\text { Quark fermion mass }}{\Psi} \cong \frac{Q_{f}}{\Psi}$

Table 2. Fitting of quark fermion and quark boson masses

\begin{tabular}{|c|c|c|}
\hline Quark & $Q_{f} c^{2}$ in $\mathrm{MeV}$ & $Q_{b} c^{2}$ in $\mathrm{MeV}$ \\
\hline Up & 4.401 & 1.945 \\
\hline Down & 9.4755 & 4.188 \\
\hline Strange & 152.5427 & 67.416 \\
\hline Charm & 1313.796 & 580.63 \\
\hline Bottom & 5287.579 & 2336.839 \\
\hline Top & 182160.18 & 80505.46 \\
\hline
\end{tabular}

Please see the following table- 2 for the obtained quark 'fermion' and 'boson' masses. The observed baryon and meson charge-mass spectrum can be generated from these mass units. Strange quark boson pair generates the neutral pion of rest energy $134.83 \mathrm{MeV}$. Obtained top quark boson rest energy is $80505 \mathrm{MeV}$ and is very close to the observed $\mathrm{W}$ boson rest energy $80.450 \pm 0.058 \mathrm{GeV}$ and 
$80.392 \pm 0.039 \mathrm{GeV}$. Please refer $M$. Yao et al[24] recommended PDG data. Really this is a great coincidence and support for the proposed new idea of "fermion-boson" unification scheme. This strongly supports super symmetry with s mall modifications.

\subsection{Beta decay, Higg's Charged Fermion and Its Boson}

It is well established that in Beta decay electron is instantaneously created from neutron and this nuclear weak force is mediated by $W$ and $Z$ bosons. If $W$ boson is really the SUSY partner of top quark then the role of $\mathrm{W}$ boson in weak decay seems to be nothing. Its role is taken up by the newly proposed Higgs charged boson of rest energy close to $45.6 \mathrm{GeV}$. Its rest energy is equal to half the rest energy of neutral $Z$ boson. Semi empirically it is noticed that

$$
\frac{m_{e} c^{2}}{F_{W} R_{0}} \cong \frac{\Psi M_{H b}}{m_{e}}
$$

Here, $M_{H b}$ is the rest mass of charged Higgs boson and $\Psi M_{H b}$ is its fermionic form. $\Psi$ is a unified SUSY fermion and boson mass ratio $=2.2627 . m_{e}$ is the rest mass of electron, $R_{0}$ is nuclear characteristic charge radius. Mass of $\Psi M_{H b}$ or $M_{H f}$ can be expressed as

$$
\begin{gathered}
M_{H f} c^{2} \cong\left(\frac{m_{e} c^{2}}{F_{W} R_{o}}\right) \cdot m_{e} c^{2} \\
M_{H b} c^{2} \cong \frac{M_{H f} c^{2}}{\Psi} \cong \frac{1}{\Psi} \cdot\left(\frac{m_{e} c^{2}}{F_{W} R_{o}}\right) \cdot m_{e} c^{2}
\end{gathered}
$$

Here accuracy depends on $R_{0}$. Based on strong nuclear gravity it was also noticed that

$$
\begin{gathered}
\hbar \cong \sqrt{\frac{G\left(N \cdot m_{e}\right)^{2} m_{e} R_{0}}{2}} \\
M_{H f} c^{2} \cong \frac{1}{2} \cdot\left(\frac{G\left(N . m_{e}\right)^{2}}{\hbar c}\right)^{2} \cdot m_{e} c^{2} \\
\cong 103125.417 \mathrm{MeV} \\
M_{H b} c^{2} \cong \frac{M_{H f} c^{2}}{\Psi} \cong \frac{1}{2 \Psi} \cdot\left(\frac{G\left(N . m_{e}\right)^{2}}{\hbar c}\right)^{2} \cdot m_{e} c^{2} \\
\cong 45576.1467 \mathrm{MeV}
\end{gathered}
$$

\subsection{Rest Energy of the Neutral $Z$ Boson}

From above estimation, neutral $Z$ boson rest energy can be given as

$$
\begin{gathered}
m_{Z} c^{2} \cong\left(M_{H b} c^{2}\right)^{ \pm}+\left(M_{H b} c^{2}\right)^{\mp} \cong 2 M_{H b} c^{2} \\
\cong 91152.293 \mathrm{MeV}
\end{gathered}
$$

This value can be compared with the experimental rest energy of $Z$ boson $=91187.621 \mathrm{MeV}[24]$.

\subsection{Recently Discovered Bos on of Rest Energy $126 \mathrm{GeV}$}

Close to the predicted rest energy of Higgs boson, recently a new boson of rest energy 124 to $160 \mathrm{GeV}$ was reported[6,7]. Surprising thing is that its existence is not matching with the current theoretical predictions. In this critical situation, with the help of strong nuclear gravity and modified super symmetry concepts, authors made an attempt to understand the origin of this new boson[3]. In our previous paper[2] it was suggested that: $W$ boson is the super symmetric boson of the top quark fermion and the charged Higgs boson pair generates the neutralized $Z$ boson.

It is noticed that Higgs charged boson and top quark boson couples together to form a new neutral boson of rest energy $126.0 \mathrm{GeV}$. This is a very interesting observation. Like $Z$ boson it can decay into 2 charged particles.

$$
\left(M_{H b} c^{2}\right)^{ \pm}+\left(m_{W} c^{2}\right)^{\mp} \cong 126.0 \mathrm{GeV}
$$

\section{Quark Baryon and Quark Meson Masses with SUSY Higgs Charged Particle}

In our earlier published paper it it was assumed that[2], if $Q_{F}$ is the quark baryon rest mass

$$
Q_{F} c^{2} \cong\left[M_{G f}^{2} \cdot Q_{f}\right]^{\frac{1}{3}} c^{2}
$$

If $Q_{E}$ is the quark effective baryon rest mass,

$$
Q_{E} c^{2} \cong\left[M_{G f}^{2} \cdot Q_{e f}\right]^{\frac{1}{3}} c^{2}
$$

If $Q_{M}$ is the quark meson rest mass,

$$
Q_{M} c^{2} \cong\left[M_{G b}^{2} \cdot Q_{b}\right]^{\frac{1}{3}} c^{2}
$$

where $M_{G f} c^{2} \cong 11460 \mathrm{MeV}$ and its bosonic form $M_{G b} c^{2} \cong \frac{M_{G f} c^{2}}{\Psi} \cong 5066 \mathrm{MeV}$.

With reference to the newly proposed Higgs charged fermion and boson[3], above relations can be expressed as

$$
\begin{aligned}
& Q_{F} c^{2} \cong x\left[M_{H f}^{2} \cdot Q_{f}\right]^{\frac{1}{3}} c^{2} \\
& Q_{E} c^{2} \cong x\left[M_{H f}^{2} \cdot Q_{e f}\right]^{\frac{1}{3}} c^{2} \\
& Q_{M} c^{2} \cong x\left[M_{H b}^{2} \cdot Q_{b}\right]^{\frac{1}{3}} c^{2} \\
& \text { where } \quad \mathrm{x} \cong \frac{1}{2 \alpha\left(\mathrm{X}_{\mathrm{E}}+1\right)} \cong 0.23143232
\end{aligned}
$$

Please see table-3 for the quark baryon rest energies and see table-4 for the quark meson rest energies. 
Table 3. Fitting of quark baryon and quark effective baryon rest energies

\begin{tabular}{|c|c|c|c|c|}
\hline Quark & $\begin{array}{c}Q_{f} c^{2} \text { in } \\
\mathrm{MeV}\end{array}$ & $\begin{array}{c}Q_{F} c^{2} \text { in } \\
\mathrm{MeV}\end{array}$ & $\begin{array}{c}Q_{e f} c^{2} \text { in } \\
\mathrm{MeV}\end{array}$ & $\begin{array}{c}Q_{E} c^{2} \text { in } \\
\mathrm{MeV}\end{array}$ \\
\hline Up & 4.401 & 834.04 & 2.456 & 686.66 \\
\hline Down & 9.4755 & 1076.97 & 5.2878 & 886.67 \\
\hline Strange & 152.5427 & 2719.35 & 85.127 & 2238.84 \\
\hline Charm & 1313.796 & 5574.13 & 733.165 & 4589.18 \\
\hline Bottom & 5287.579 & 8866.53 & 2950.74 & 7299.81 \\
\hline Top & 182160.18 & 28850.43 & 101654.72 & 23752.56 \\
\hline
\end{tabular}

Table 4. Fitting of quark boson and quark meson rest energies

\begin{tabular}{|c|c|c|}
\hline Quark & $Q_{b} c^{2}$ in $\mathrm{MeV}$ & $Q_{M} c^{2}$ in $\mathrm{MeV}$ \\
\hline Up & 1.945 & 368.6 \\
\hline Down & 4.188 & 475.98 \\
\hline Strange & 67.416 & 1201.81 \\
\hline Charm & 580.63 & 2463.48 \\
\hline Bottom & 2336.839 & 3918.55 \\
\hline Top & 80505.46 & 12750.41 \\
\hline
\end{tabular}

\subsection{Rest energy of the Nucleon}

From table-3 it is noticed that, nucleon mass is very close to the harmonic mean of the up baryon and down baryon masses.

$$
\frac{2\left(u_{F} c^{2}\right)\left(d_{F} c^{2}\right)}{\left(u_{F}+d_{F}\right) c^{2}} \cong 940.06 \mathrm{MeV}
$$

where $u_{F} c^{2} \cong 834.04 \mathrm{MeV}$ and $d_{F} c^{2} \cong 1076.97 \mathrm{MeV}$. It is also noticed that,

$$
\left(m_{n}-m_{p}\right) c^{2} \cong \sin ^{2} \theta_{W}\left[\frac{2\left(u_{f} c^{2}\right)\left(d_{f} c^{2}\right)}{\left(u_{f}+d_{f}\right) c^{2}}\right] \cong 1.2964 \mathrm{MeV}
$$

where $m_{p}$ and $m_{n}$ are the rest masses of proton and neutron respectively.

\subsection{Super Fine Levels of $b \bar{b}$ Neutral Mesons}

In this model no 2 baryons couples together to form a neutral meson. Please note that only bottom meson and anti bottom meson couples together to form a neutral meson. The ground state rest energy is equal to $2 * 3918.55=7837.1$ $\mathrm{MeV}$. It's super fine levels can be expressed as $\left[\frac{n(n+1)}{2}\right]^{\frac{1}{12}} \cdot 7837.1 \mathrm{MeV}$ where $n=1,2,3 \ldots$ Please see the following table-5.

In this table at $n=8$ obtained rest energy is 10564.46 $\mathrm{MeV}$ and can be compared with the newly discovered $b \bar{b}$ meson. It can be suggested that, average of any two successive levels can also be seen as an excited state. In the similar way excited levels of $c \bar{c}$ and $t \bar{t}$ can be understood[2]. Not only that, for the integral charge quark model, $b \bar{u}, b \bar{d}, b \bar{s}, b \bar{c}$, etc excited levels can be predicted. With the same idea $c \bar{u}, c \bar{d}$ and $c \bar{s}$ excited levels can also be predicted and fitted[2].

Table 5. Fitting of excited levels $b \bar{b}$ meson rest energies

\begin{tabular}{|c|c|c|}
\hline$n$ & {$\left[\frac{n(n+1)}{2}\right]$} & {$\left[\frac{n(n+1)}{2}\right]^{\frac{1}{12}} 7837.1$} \\
\hline 1 & 1 & 7837.1 \\
\hline 2 & 3 & 8588.46 \\
\hline 3 & 6 & 9099.16 \\
\hline 4 & 10 & 9494.86 \\
\hline 5 & 15 & 9821.16 \\
\hline 6 & 21 & 10100.44 \\
\hline 7 & 28 & 10345.51 \\
\hline $\mathbf{8}$ & $\mathbf{3 6}$ & $\mathbf{1 0 5 6 4 . 4 6}$ \\
\hline 9 & 45 & 10762.74 \\
\hline 10 & 55 & 10944.24 \\
\hline
\end{tabular}

\subsection{To Fit the Size of Proton}

It is noticed that,

$$
R_{p} \cong \sqrt{\frac{e^{2}}{4 \pi \varepsilon_{0} G m_{p}^{2}}} ? \frac{2 G\left(N . m_{e}\right)}{c^{2}} \cong .90566 \mathrm{fm}
$$

This obtained magnitude can be compared with the rms charge radius of the proton. With different experimental methods its magnitude varies from $0.84184(67) \mathrm{fm}$ to $0.895(18) \mathrm{fm}[8,26,27]$. Here it is very interesting to consider the role of the Schwarzschild radius of the 'molar electron mass'. From reference[25], $R_{p}=0.870 \pm 0.023 \pm 0.012 \mathrm{fm}$. This type of coincidence can not be ignored in the unification scheme.

\subsection{Scattering Distance between Electron and the Nucleus}

If $R_{0} \cong 1.21$ to $1.22 \mathrm{fm}$ is the minimum scattering distance between electron and the nucleus, it is noticed that,

$$
R_{0} \cong\left(\frac{\hbar c}{G\left(N m_{e}\right)^{2}}\right) \cdot\left(\frac{\hbar c}{G m_{e}^{2}}\right) \cdot \frac{2 G m_{e}}{c^{2}} \cong 1.21565 \mathrm{fm}
$$

Here $\left(N m_{e}\right)$ is the molar electron mass. Here also it is very interesting to consider the role of the Schwarzschild radius of the 'electron mass'. Thus the two macroscopic physical constants $N$ and $G$ can be expressed in the following way.

$$
\begin{array}{r}
N \cong \sqrt{\frac{2 \hbar^{2}}{G m_{e}^{3} R_{0}}} \\
G \cong \frac{2 \hbar^{2}}{\left(N m_{e}\right)^{2} m_{e} R_{0}}
\end{array}
$$

In this way, either the Avogadro number or the 
gravitational constant can be obtained.

\subsection{Electromagnetic and Gravitational Force Ratio of the Proton, Proton-electron Mass Ratio and the Squared Avog adro Number}

Semi empirically it is noticed that

$$
\ln \sqrt{\frac{e^{2}}{4 \pi \varepsilon_{0} G m_{p}^{2}}} \cong \sqrt{\frac{m_{p}}{m_{e}}-\ln \left(\frac{G\left(N . m_{e}\right)^{2}}{G m_{e}^{2}}\right)}
$$

where $m_{p}$ is the proton rest mass and $m_{e}$ is the electron rest mass. Thus

$$
\ln \sqrt{\frac{e^{2}}{4 \pi \varepsilon_{0} G m_{p}^{2}}} \cong \sqrt{\frac{m_{p}}{m_{e}}-\ln \left(N^{2}\right)}
$$

Considering this as a characteristic relation, proton rest mass can be fitted accurately in the following way.

$$
\left(e^{\sqrt{\frac{m_{p}}{m_{e}}-\ln \left(N^{2}\right)}}\right)^{2} m_{p}^{2} \cong \frac{e^{2}}{4 \pi \varepsilon_{0} G} .
$$

The gravitational constant can be expressed as

$$
G \cong\left(e^{\sqrt{\frac{m_{p}}{m_{e}}-\ln \left(N^{2}\right)}}\right)^{-2} ? \frac{e^{2}}{4 \pi \varepsilon_{0} m_{p}^{2}}
$$

Thus $G \cong 6.666270179 \times 10^{-11} \mathrm{~m}^{3} \mathrm{Kg}^{-1} \mathrm{sec}^{-2}$.

Avogadro number can be expressed as

$$
N \cong \sqrt{\exp \left[\frac{m_{p}}{m_{e}}-\left(\ln \sqrt{\frac{e^{2}}{4 \pi \varepsilon_{0} G m_{p}^{2}}}\right)^{2}\right]}
$$

Thus $N \cong 6.174407621 \times 10^{23}$.

\section{To Fit the Semi Empirical Mass Formula Energy Coefficients}

The semi-empirical mass formula (SEMF) is used to approximate the mass and various other properties of an atomic nucleus[28,29]. As the name suggests, it is based partly on theory and partly on emp irical measurements. The theory is based on the liquid drop model proposed by George Gamow and was first formulated in 1935 by German physicist Carl Friedrich von Weizsacker. Based on the 'least squares fit', volume energy coefficient is $a_{v}=15.78 \mathrm{MeV}$, surface energy coefficient is $a_{s}=18.34$ $\mathrm{MeV}$, coulombic energy coefficient is $a_{c}=0.71 \mathrm{MeV}$, asymmetric energy coefficient is $a_{a}=23.21 \mathrm{MeV}$ and pairing energy coefficient is $a_{p}=12 \mathrm{MeV}$. The semi emp irical mass formula is

$$
B E \cong A a_{v}-A^{\frac{2}{3}} a_{s}-\frac{Z(Z-1)}{A^{\frac{1}{3}}} a_{c}-\frac{(A-2 Z)^{2}}{A} a_{a} \pm \frac{1}{\sqrt{A}} a_{p}
$$

In a unified approach it is noticed that, the energy coefficients are having strong inter-relation with the proton rest mass and the 'mole electron mass'. The interesting observations can be expressed in the following way.

\subsection{The Coulombic Energy Coefficient}

It can be defined as,

$$
a_{c} \cong \alpha \text { 詼 } 0 m 6{ }_{p}^{2} 1 \mathrm{eV}
$$

Ratio of the coulomb ic energy coefficient and the proton rest energy is close to the product of the fine structure ratio and the strong coupling constant.

\subsection{The Surface and Volume Energy Coefficients}

Surface energy coefficient can be defined as

$$
a_{s} \cong \sqrt{\frac{G\left(N . m_{e}\right)^{2}}{\hbar c}} a_{c} \cong 9.36 \mathrm{MeV}
$$

Volume energy coefficient can be defined as

$$
\begin{gathered}
a_{v} \cong \sqrt{\frac{G\left(N \cdot m_{e}\right)^{2}}{\sqrt{2} \hbar c}} a_{c} \cong 6.28 \mathrm{MeV} \\
\text { Thus, } \frac{\mathrm{a}_{\mathrm{s}}}{\mathrm{a}_{\mathrm{v}}} \cong 2^{\frac{1}{4}}
\end{gathered}
$$

\subsection{The Asymmetry and Pairing Energy Coefficients}

Asymmetry energy coefficient can be defined as

$$
a_{a} \cong \frac{2}{3}\left(a_{v}+a_{s}\right) \cong 23.76 \mathrm{MeV}
$$

Pairing energy coefficient is close to

$$
a_{p} \cong \frac{1}{3}\left(a_{v}+a_{s}\right) \cong 11.88 \mathrm{MeV}
$$

Thus, $a_{v}+a_{s} \cong a_{a}+a_{p} \cong 35.64 \mathrm{MeV}$

In table- 6 considering the magic numbers, within the range of $(Z=26 ; A=56)$ to $(Z=92 ; A=238)$ nuclear binding energy is calculated and compared with the measured binding energy[30]. Column-3 represents the calculated binding energy and column-4 represents the measured binding energy. If th is procedure is found to be true and valid then with a suitable fitting procedure qualitatively and quantitatively magnitudes of the proposed SEMF binding energy coefficients can be refined.

Table 6. SEMF binding energy with the proposed energy coefficients

\begin{tabular}{|c|c|c|c|c|}
\hline$Z$ & $A$ & $(B E)_{c a l}$ in MeV & $\begin{array}{c}(B E)_{\text {mes }} \text { in } \\
\mathrm{MeV}\end{array}$ & \% Error \\
\hline 26 & 56 & 492.60 & 492.254 & -0.0713 \\
\hline 28 & 62 & 547.08 & 545.259 & -0.335 \\
\hline 34 & 84 & 728.29 & 727.341 & -0.131 \\
\hline 50 & 118 & 1007.46 & 1004.950 & -0.250 \\
\hline 60 & 142 & 1183.64 & 1185.145 & 0.127 \\
\hline 79 & 197 & 1554.82 & 1559.40 & 0.293 \\
\hline 82 & 208 & 1625.22 & 1636.44 & 0.686 \\
\hline 92 & 238 & 1803.12 & 1801.693 & -0.0795 \\
\hline
\end{tabular}




\subsection{Proton-nucleon Stability}

It is noticed that

$$
\frac{A_{s}}{2 Z} \cong 1+2 Z\left(\frac{a_{c}}{a_{s}}\right)^{2} \cong 1+2 Z\left(\frac{\hbar c}{G\left(N . m_{e}\right)^{2}}\right)
$$

where $A_{s}$ is the stable mass number of $Z$. This is a direct relation. Assuming the proton number $Z$, in general, for all atoms, lower stability can be fitted directly with the following relation[28].

$$
\begin{aligned}
& A_{s} \cong 2 Z\left[1+2 Z\left(\frac{a_{c}}{a_{s}}\right)^{2}\right] \cong 2 Z+Z^{2} * 0.0063 \\
& Z=21, A_{s} \cong 44.78 ; \text { if } Z=29, A_{s} \cong 63.29 ;
\end{aligned}
$$

if $Z=47, A_{s} \cong 107.91$; if $Z=53, A_{s} \cong 123.68$;

if $Z=60, A_{s} \cong 142.66$; if $Z=79, A_{s} \cong 197.29$;

if $Z=83, A_{s} \cong 209.37$; if $Z=92, A_{S} \cong 237.29$;

Stable super heavy elements can be predicted with this relation. In between $Z=30$ to $Z=60$ obtained $A_{s}$ is lower compared to the actual $A_{s}$. It is noticed that, upper stability in light and medium atoms up to $Z \approx 56$ can be fitted with the following relation.

$$
\begin{gathered}
A_{s} \cong 2 Z\left[1+2 Z\left(\left(\frac{a_{c}}{a_{s}}\right)^{2}+\left(\frac{a_{c}}{a_{a}+a_{p}}\right)^{2}\right)\right] \\
\cong 2 Z+Z^{2} * 0.0082
\end{gathered}
$$

From this relation for $Z=56$, obtained upper $A_{s} \cong 137.7$. Note that, for $Z=56$, actual stable $A_{s} \cong 137 \cong \frac{1}{\alpha}$ where $\alpha$ is the fine structure ratio. This seems to be a nice and interesting coincidence. In between 0.0063 and 0.0082 , for light and medium atoms up to $Z \approx 56$ or $A_{s} \approx 137$, mean stability can be fitted with the following relation.

$$
A_{s} \cong 2 Z+Z^{2} * 0.0072
$$

Surprisingly it is noticed that, in this relation, $0.0072 \approx \alpha \cong 0.0073$. Thus up to $Z \cong 56$ or $A_{s} \approx 137$, mean stability can be expressed as

$$
A_{s} \approx 2 Z+\left(Z^{2} \alpha\right)
$$

\subsection{To fit and Predict the Magic Numbers}

After 1932, experimental data on nuclei repeatedly revealed that in the nucleus, there exists a series of magic numbers, called so, because nuclei with these values for the atomic number $Z$ and/or neutron number $N$, showed exceptional stability[31,32]. The observed magic numbers are: $2,8,20,28,50,82$ and 126 . The separation energy for one proton or one neutron is very large for a nucleus with $Z$ or $N$ magic. The probability of nuclei with neutron numbers $20,28,50,82$ or 126 capturing a neutron is much less than that of their neighbouring nuclei.
Considering $\alpha$ as the characteristic stability number, in a reverse order, magic numbers $82,50,28,20$ and 8 can be fitted[33]. Data indicates that for heavy elements stability is achieved when neutron-proton ratio approaches $\approx 1.6$. Let us define a new number $k$ in the following way:

$$
k \cong\left(\frac{m_{n}-m_{p}}{2 m_{e}}\right)^{2}-1 \cong 0.6014
$$

Magic numbers can be fitted with the following semi emp irical relation.

$$
M_{n} \cong\left[k^{n} \cdot\left(\frac{1}{\alpha}\right)\right]+\left[(-1)^{n} \cdot(n \cdot k)\right]
$$

where $n \cong 1,2,3,4,5$ and 6 . Thus $\quad M_{n} \cong$ $81.81,50.77,28.0,20.33,7.77$ and 10.09. After rounding off the values to the nearest even integers, we get, $82,50,28,20,8$ and 10. At $\mathrm{n}=7 M_{n} \cong-0.31$ and thus the series seems to be closed at $\mathrm{n}=6$ itself. Here magic numbers 2 and 126 are missing. The predicted new light magic number is 10 . The proposed expression is very simple. It clearly indicates that $-\alpha$ is strongly interconnected with nuclear closed shells.

Heavy magic numbers above and below 126 can be predicted with the following expression.

$$
M_{n} \approx\left[1 \pm \alpha^{\frac{1}{n}}\right] \cdot\left(\frac{1}{\alpha}\right)
$$

For, $\left(1+\alpha^{\frac{1}{n}}\right)$ series, $M_{n} \approx$

138.04, 148.74, 163.62, 177.09, 188.26, 197.38,

For, $\left[1-\alpha^{\frac{1}{n}}\right]$ series, $M_{n} \approx$

136.04, 125.33,110.46,96.98,85.81,76.68,

Fine tuning can be achieved if it is assumed that

$$
M_{n} \cong\left[\left(1 \pm \alpha^{\frac{1}{n}}\right) \cdot\left(\frac{1}{\alpha}\right)\right]+\left[(-1)^{n} \cdot(n \cdot k)\right]
$$

For, $\left[1+\alpha^{\frac{1}{n}}\right]$ series, $M_{n} \cong 137.43,149.94,161.18,179.49$, 185.25, 200.99,

For, $\left[1-\alpha^{\frac{1}{n}}\right]$ series, $M_{n} \cong 135.43,126.53,108.65,99.39$, 82.80, 80.29,

After rounding off the values to the nearest even integers, we get,

$$
\begin{aligned}
& M_{n} \cong 136,150,160,178,184,200, \text { and } \\
& M_{n} \cong 134,126,108,98,82,80,
\end{aligned}
$$

Magic number 184 can be observed in $\left[1+\alpha^{\frac{1}{n}}\right]$ series and 126 can be observed in $\left[1-\alpha^{\frac{1}{n}}\right]$ series. 


\subsection{Nuclear Binding Energy with 2 Terms and Only one Energy Constant}

Nuclear binding energy can be fitted with 2 terms or 4 factors with $a_{c} \cong 0.7681 \mathrm{MeV}$ as the single energy constant. First term can be expressed as

$$
T_{1} \cong(f)(A+1) \ln \left[(A+1) X_{S}\right] a_{c}
$$

where $f \cong 1+\frac{2 Z}{A_{S}} \leq 2.0$ and $X_{S} \cong 8.91424$ is the strong coupling constant.

Second term can be expressed as

$$
T_{2} \cong\left[\frac{A^{2}+\left(f \cdot Z^{2}\right)}{X_{S}^{2}}\right] a_{c}
$$

Close to the stable mass number $A_{s}$,

$$
\text { B. } E=T_{1}-T_{2}
$$

Please see the following data.

$Z=2 \quad \& \quad A=4, B . E \cong 28.93 \mathrm{MeV}$;

$Z=10 \quad \& \quad A=20, B . E \cong 160.44 \mathrm{MeV}$;

$Z=26 \quad \& \quad A=56, B . E \cong 482.06 \mathrm{MeV}$;

$Z=50 \& A=118, B . E \cong 1007.35 \mathrm{MeV}$;

$Z=79 \& A=197, B . E \cong 1563.72 \mathrm{MeV}$;

$Z=82 \& A=208, B . E \cong 1634.76 \mathrm{MeV}$;

$Z=92 \& A=238, B . E \cong 1805.15 \mathrm{MeV}$.

Above 2 terms can be put into 4 factors as

$$
B . E \cong\left[2-\frac{A}{2 Z}\right](f)(A+1) \ln \left[(A+1) X_{S}\right] a_{c}
$$

With this relation,

$Z=2 \quad \& \quad A=4, B . E \cong 29.07 \mathrm{MeV}$;

$Z=10 \quad \& \quad A=20, B . E \cong 160.98 \mathrm{MeV}$;

$Z=26 \& A=56, B . E \cong 484.56 \mathrm{MeV}$;

$Z=50 \& A=118, B . E \cong 973.32 \mathrm{MeV}$

$Z=79 \& A=197, B . E \cong 1542.1 \mathrm{MeV}$

$Z=82 \& A=208, B . E \cong 1587.52 \mathrm{MeV}$;

$Z=92 \& A=238, B . E \cong 1764.8 \mathrm{MeV}$;

These relations can be considered for further research and analysis positively.

\section{Magnetic Moments of the Nucleon}

If $\left(\alpha X_{E}\right)^{-1} \cong \sin \theta_{W}$, magnetic moment of electron can be expressed as

$$
\mu_{e} \cong \frac{1}{2} \sin \theta_{W} \cdot e c \cdot \sqrt{\frac{e^{2}}{4 \pi \varepsilon_{0} F_{W}}} \cong 9.274 \times 10^{-24} \mathrm{~J} / \text { tesla }
$$

It can be suggested that electron's magnetic moment is due to the nuclear weak force. Similarly magnetic moment of proton is due to the nuclear strong force and is close to

$$
\mu_{p} \cong \frac{1}{2} \sin \theta_{W} \cdot e c \cdot \sqrt{\frac{e^{2}}{4 \pi \varepsilon_{0} F_{S}}}
$$

where $R_{0} \cong 1.21565 \mathrm{fm}$ and $F_{S} \cong \frac{e^{2}}{4 \pi \varepsilon_{0} R_{0}^{2}} \cong 156.115$ newton is the strong force magnitude. Thus

$$
\mu_{p} \cong \frac{1}{2} \sin \theta_{W} \cdot e c \cdot R_{0} \cong 1.356 \times 10^{-26} \mathrm{~J} / \text { tesla }
$$

If proton and neutron are the two quantum states of the nucleon, by considering the radius of proton $R_{p}$, magnetic moment of neutron can be fitted as

$$
\mu_{n} \cong \frac{1}{2} \sin \theta_{W} \cdot e c \cdot R_{p} \cong 9.782 \times 10^{-27} \mathrm{~J} / \text { tesla }
$$

\section{Mystery of the Gram Mole}

If $M_{P} \cong \sqrt{\frac{\hbar c}{G}}$ is the Planck mass and $m_{e}$ is the rest mass of electron, semi empirically it is observed that,

$$
\begin{gathered}
M_{g} \cong N^{-\frac{1}{3}} \sqrt{\left(N \cdot M_{P}\right)\left(N \cdot m_{e}\right)} \cong 1.0044118 \times 10^{-3} \mathrm{Kg} \\
M_{g} \cong N^{\frac{2}{3}} \cdot \sqrt{M_{P} m_{e}}
\end{gathered}
$$

Here $M_{g}$ is just crossing the mass of one gram. If $m_{p}$ is the rest mass of proton,

$$
\begin{gathered}
\frac{M_{g}}{m_{p}} \cong \frac{N^{\frac{2}{3}} \cdot \sqrt{M_{P} m_{e}}}{m_{p}} \cong N \cong 6.003258583 \times 10^{23} \\
\text { Thus } \frac{\sqrt{\mathrm{M}_{\mathrm{P}} \mathrm{m}_{\mathrm{e}}}}{\mathrm{m}_{\mathrm{p}}} \cong \mathrm{N}^{\frac{1}{3}}
\end{gathered}
$$

Obtained $N \cong 5.965669601 \times 10^{23}$. More accurate empirical relation is

$$
\frac{\sqrt{M_{P} m_{e}} c^{2}}{\left[\frac{m_{p} c^{2}+m_{n} c^{2}-B_{a}}{2}+m_{e} c^{2}\right]} \cong N^{\frac{1}{3}}
$$

where $m_{n}$ is the rest mass of neutron, and $B_{a} \approx 8 \mathrm{MeV}$ is the mean binding energy of nucleon. Obtained value of $N \cong 6.020215677 \times 10^{23}$.

\section{Conclusions}

Here it is very important to understand the role of the proposed 'molar electron mass'. If it represents and indicates the existence of a massive heavy charged particle, automatically Avogadro number can be eliminated. It is having multiple applications in particle physics and cosmology. Authors are working in this new direction. According to Hawking in particular, 'M-theory is the only candidate for a complete theory of the universe'. Nevertheless physicists, such as Feynman and Glashow, have criticized string theory for not providing novel 
experimental predictions at accessible energy scales. The success of any TOE depends on - how it couples the Planck scale and the nuclear scale. Please note that, in its initial stage, string theory was originated for understanding the strong interaction. In this connection, by coupling the proposed ideas with the basic concepts of String theory or Super gravity, basic ideas of unification can be refined. Not only that Planck scale and nuclear scale can be interlinked. Further research, analysis and experimental data may reveal the facts.

\section{ACKNOWLEDGEMENTS}

The first author is indebted to professor K. V. Krishna Murthy, Chairman, Institute of Scientific Research on Vedas (I-SERVE), Hyderabad, India and Shri K. V. R. S. Murthy, former scientist IICT (CSIR) Govt. of India, Director, Research and Development, I-SERVE, for their valuable guidance and great support in developing this subject.

\section{REFERENCES}

[1] G. Aad et al. (ATLAS Collaboration), Rev. Lett. 108 pp 15 2012

[2] U. V. S. Seshavatharam and S. Lakshminarayana, Int. J. Mod. Phys. E, 19, No.2, pp.263-280, 2010.

[3] U. V. S. Seshavatharam and S. Lakshminarayana, Hadronic journal, 34, No 3, June, pp277-300, 2011.

[4] U. V. S. Seshavatharam and S. Lakshminarayana, Proceedings of the DAE Symp. on Nucl. Phys. 56 pp.302, 2011.

[5] U. V. S. Seshavatharam and S. Lakshminarayana, Proceedings of the DAE Symp. on Nucl. Phys. 56 pp.842, 2011.

[6] T. Aaltonen et al, Phys.Rev.Lett.106:pp 171801, 2011.

[7] Ning Chen, Hong-Jian He, http://arxiv.org/abs/1202.3072

[8] P. J. Mohr and B.N. Taylor, http://physics.nist.gov/constants.

[9] Halpern, Paul, Hoboken, New Jersey: John Wiley Sons, Inc.. pp. 326. ISBN 0-471-46595-X, 2011.

[10] Smolin, Lee, New York: Houghton Mifflin Co.. pp. 392. ISBN 0-618-55105-0, 2006.

[11] Woit, Peter, London: Jonathan Cape \&: New York: Basic
Books. pp. 290. ISBN 978-0-465-09275-8, 2006.

[12] E. Cremmer, B. Julia and J. Scherk, Physics Letters B76 pp 409-412, 1978.

[13] Wess, Julius; Bagger, Jonathan, Princeton University Press. pp. 260. ISBN 0691025304, 1992.

[14] Abdus Salam, NATO Advanced Study Institute, Erice, June16-July 6, 1972.

[15] Salam A, Sivaram C, Mod. Phys. Lett., v. A8(4), pp.321-326, 1993.

[16] Recami E. Preprint NSF-ITP-02-04. posted in the arXives as the e-print physics $/ 0505149$, and references therein.

[17] P. Caldirola, M. Pavsic and Recami E, IL Nuovo Cimento . 48 B, No. 2, 11 Dec 1978.

[18] Recami E and V. T. Zanchin, Foundations of Physics letters, 7, no.1, 1994.

[19] V. T. Zanchin and Recami E, Foundations of Physics letters, 7, no.2, 1994.

[20] S. I. Fisenko, M. M. Beilinson and B. G. Umanov, Physics Letters A, 148, Issues 8-9, pp 405-407, 1990.

[21] Fedosin S.G, Journal of Vectorial Relativity, 4, No. 1, pp.124, 2009.

[22] U. V. S. Seshavatharam and S. Lakshminarayana, Hadronic journal, 34, No 4, Aug. p379, 2011.

[23] U. V. S. Seshavatharam, Progress in Physics, 2, pp.7-14, 2010.

[24] Particle Data Group (W.-M. Yao et al.), J. Phys. G 33 (2006) 1, http://pdg.bbb.gov.

[25] S. Atashbar Tehrani and A. N. Khorramian, CPC (HEP \& NP), 34(9): pp 1485-1487. 2010.

[26] Richard J. Hill and Gil Paz, Phys.Rev.D82, pp 113005, 2010.

[27] Michael O. Distler et al, Phys. Lett. B696: pp.343-347, 2011.

[28] P. Roy Chowdhury et al, Mod. Phys. Lett. A20 pp.16051618, 2005.

[29] W.D. Myers and W.J. Swiatecki, LBL-36803, nsdssd.lbl.gov.

[30] G. Audi and A.H. Wapstra. Nuclear physics, A 565, pp.1-65. 1993 ,

[31] R Velusamy, Resonance, pp 12-24, December 2007.

[32] REN Zhong-Zhou et al, Commun. Theor. Phys. (Beijing, China) 42 pp. 851- 866, 42, No. 6, December 15, 2004.

[33] U. V. S. Seshavatharam and S. Lakshminaray ana, Hadronic journal. 35, No. 1, pp.113, 2012. 